\title{
Combined Treatment of Bone Marrow Mesenchymal Stem Cells And Fasudil Promotes Neurovascular Remodeling And Neurological Function Recovery In Ischemic Stroke
}

Qian Wang ( $\square$ wangqian881218@sina.com )

Dalian Medical College: Dalian Medical University

Shu-Fang Zhao

Dalian Medical College: Dalian Medical University

Xia Xiao

Dalian Medical College: Dalian Medical University

Ya-Nan Liu

Dalian Medical College: Dalian Medical University

\section{Xiu Li Wang}

Dalian Medical College: Dalian Medical University

\section{Yun-Xia Du}

Dalian Medical College: Dalian Medical University

\section{Research Article}

Keywords: bone marrow mesenchymal stem cells, fasudil, ischemic stroke, neurological function, inflammation, infarction

Posted Date: July 20th, 2021

DOI: https://doi.org/10.21203/rs.3.rs-720071/v1

License: (c) (i) This work is licensed under a Creative Commons Attribution 4.0 International License. Read Full License

Version of Record: A version of this preprint was published at Applied Biochemistry and Biotechnology on September 20th, 2021. See the published version at https://doi.org/10.1007/s12010-021-03679-6. 


\section{Abstract}

Stroke remains a deadly and disabling disease with limited treatment tragedies due to the limitations of available treatments; novel therapies for stroke are needed. In this article, the synergistic results of dual bone marrow mesenchymal stem cells (BMSC) and fasudil treatment in rat models of ischemic stroke still requires further identification. Sprague-Dawley rats were used to construct the middle cerebral artery, occlusion models. BMSCs were incubated with fasudil, and MTT was performed to evaluate cell proliferation. The rats were treated with fasudil+BMSC, BMSC, fasudil, and saline. Blood samples were collected for complete blood count analysis and measurement of serum TNF-a levels. The neurological functions were evaluated. After the rats were sacrificed, immunohistochemical staining and TTC staining was performed. Fasudil promoted the proliferation of BMSCs and induced their differentiation into neuron-like cells. BMSCs increased the proportion of neutrophils; nevertheless, fasudil counteracted the neutrophil increase. The TUJ-1/MAP2/VIII factor expression in the fasudil+BMSC group was significantly higher than that in the other groups. The number of GFAP-positive cells decreased in the fasudil+BMSC and BMSC alone groups. The infarct volume in the fasudil+BMSC and BMSC alone groups was significantly lower than in the fasudil alone and control groups.Both BMSCs and fasudil exert neurorestorative effects in rat models of cerebral ischemia. Fasudil neutralizes the pro-inflammatory effects of BMSCs, while BMSCs and fasudil together had synergistic effects promoting neurovascular remodeling and neurological function recovery in stroke. A combination of BMSCs and fasudil provides a promising method for the treatment of ischemic stroke.

\section{Introduction}

Stroke is currently the second leading cause of death worldwide and results in most disability cases [1]. Antithrombosis, the mainstream treatment for ischemic stroke, has contributed significantly to the improvement of clinical prognosis. Nevertheless, antithrombotic agents require strict indications for administration, limiting the patient pool to a small percentage of those with ischemic stroke [2]. Recently, stem cell transplantation therapy is emerging as an alternative approach to recovering the structure and functions of the central nervous system with various diseases, such as multiple sclerosis, neuromyelitis optica, etc. [3]. Autologous bone marrow mesenchymal stem cells (BMSCs) exert neuroprotective effects against cerebral ischemia, which may be mediated by autophagy inhibition or an anti-apoptotic mechanism [4, 5]. Moreover, BMSCs can be harvested from the patients themselves, avoiding ethical or immunologic repercussions.

BMSC transplantation therapy has been proven effective in numerous preclinical experiments, exhibiting neurogenesis, angiogenesis and enhanced neural plasticity in animal cerebral ischemia models [6-8]. Furthermore, several clinical trials have demonstrated the safety and feasibility of BMSC transplantation in patients with ischemic stroke [9].

Rho and its downstream effector, the Rho-associated coiled-coil protein kinase (ROCK), constitute an essential pathway regulating the microvascular structure and blood-brain barrier (BBB) permeability. 
Cumulative evidence has indicated an up-regulation of the Rho/ROCK pathway in ischemic stroke, and ROCK as a potential therapeutic target for ischemia [10]. ROCK inhibitors can reduce the infarct size and improve the functional outcome in patients with focal cerebral ischemia [11]. Chiba et al. reported that fasudil enhanced the neuroprotective effects of BMSC on promoting axon regeneration in rats with spinal cord injury [12]. The current study aimed to investigate the synergistic effects of BMSCs and fasudil in animal models of ischemic stroke.

\section{Material And Methods}

\section{Ethics statements and BMSC preparation}

This study has been reviewed and approved by the Animal Care and Use Committee of Dalian Medical University, China. BMSCs were isolated from 8-week-old male green fluorescence protein (GFP)-transgenic rats (Cyagen Biosciences Inc., Guangzhou, China) and were cultured as previously reported [13]. Cells were cultured with Dulbecco's modified eagle medium (DMEM) containing 10\% FBS, $1 \%$ nonessential amino acids, $1 \%$ glutamine, and $1 \%$ pen-strep, and they were passaged $4-5$ times before use in the following studies.

\section{Construction of rat cerebral ischemia model}

Adult male Sprague-Dawley rats (275-300 grams) were used to construct the middle cerebral artery occlusion (MCAO) models. Anesthesia was induced by inhalation of $4 \%$ isoflurane in $\mathrm{N}_{2} \mathrm{O} / \mathrm{O}_{2}(70: 30)$ and maintained with $1.5 \%$ isoflurane in $\mathrm{N}_{2} \mathrm{O} / \mathrm{O}_{2}$ (70:30). Throughout the procedure and during recovery, body temperature was maintained at $37^{\circ} \mathrm{C}$ using heating pads. The rats were shaved, the surgical site was scrubbed with Betadine and $70 \%$ ethyl alcohol. The rat was placed in a supine position, a 3-0 nylon suture with its tip coated by silicone rubber was inserted into the external carotid artery (ECA) through a small puncture. It was then advanced from the ECA to the lumen of the internal carotid artery (ICA) until the suture blocked the origin of the middle cerebral artery; the length of the nylon suture was $18-20 \mathrm{~mm}$ from the inserted position. The nylon filament was retained for $2 \mathrm{hrs}$ inside the ICA, and the neck incision was closed. The experimental rats were randomly divided into the four groups: fasudil+BMSC group $(n=20)$, BMSC alone group $(n=20)$, fasudil alone group $(n=20)$, and control group $(n=20)$. BMSC and/or fasudil were administered $24 \mathrm{~h}$ after the MCAO operation. Rats of the sham group $(n=5)$ underwent all the surgical procedures except the ligation.

\section{MTT assay}

BMSCs were incubated with various concentrations of fasudil $(10 \mathrm{umol} / \mathrm{L}, 30 \mathrm{umol} / \mathrm{L}, 50 \mathrm{umol} / \mathrm{L}, 100$ umol/L, $200 \mathrm{umol} / \mathrm{L}$, and $300 \mathrm{umol} / \mathrm{L})$ for 24 hours. Then, they were analyzed using 3-(4,5dimethylthiazol-2-yl)-2,5-diphenyltetrazolium bromide (MTT) assay according to the manufacturer's instructions. The optical density (OD) value was measured at $490 \mathrm{~nm}$ using a microplate reader (Labsystems Multiskan MS, Finland). 
In the fasudil+BMSC group, fasudil was intraperitoneally injected at $10 \mathrm{mg} / \mathrm{kg}$ and $5 \mathrm{mg} / \mathrm{kg}$, then, BMSCs $\left(3 \times 10^{6}\right.$ cells in $\left.1 \mathrm{ml} \mathrm{PBS}\right)$ were transplanted through the tail vein. In the BMSC alone group, saline was intraperitoneally injected and $3 \times 10^{6}$ BMSCs were transplanted. In the fasudil alone group, fasudil was intraperitoneally injected and saline was administered through the tail vein. In the control group, saline was administered both intraperitoneally and via the tail vein. There was no intervention in the Sham group.

Blood cell analysis

The blood samples were collected from the rats ( $n=3$ each group) via the tail vein at 3,5 , and 7 days after the BMSC/fasudil/saline treatment, using EDTA tubes (Fischer Scientific Cat NO. 22-040-101). The blood samples were kept at $4^{\circ} \mathrm{C}$ and sent to the BioReliance Corporation (Rockville, MD, USA) for complete blood count analysis.

Assessment of neurological function

A modified Neurological Severity Score (mNSS) was used to evaluate the neurological functions following MCAO at various time points: 1 day, 1 week, 2 weeks, 3 weeks, 4 weeks, 5 weeks, 6 weeks, and 7 weeks. The mNSS scale included four domains: motor, sensory, reflex, and balance. A high score (maximum, 18 points) indicated more severe cerebral damage. The assessments were performed at regular intervals by a blind evaluator.

\section{Serum TNF-a levels}

Serum TNF-a levels were measured using ELISA Quantikine Kits (Lab systems Multiskan MS, Finland) according to the manufacturer's instructions. Standard curves and samples were analyzed in triplicate, and means were calculated.

\section{Immunohistochemistry}

The rats were sacrificed 8 weeks after the MCAO operation. After being anesthetized with $4.0 \%$ isoflurane in $\mathrm{N}_{2} \mathrm{O} / \mathrm{O}_{2}(70: 30)$, the rats were transcardially perfused with saline and $4 \%$ paraformaldehyde. The brain sample was immersed in $4 \%$ paraformaldehyde for 2 days and embedded in an optimal cutting temperature compound (OCT). Then, the sample at the level of the striatum was cut into equally spaced coronal sections $(4 \mu \mathrm{m})$ for subsequent immunohistochemical staining. The slides were blocked and permeabilized in $0.1 \mathrm{M}$ phosphate buffer saline (PBS) with $0.3 \%$ Triton X-100 (Sigma Chemical Co., MO, USA) and $1 \%$ bovine serum albumin (BSA) for $1 \mathrm{~h}$. Each sample was treated with primary antibody against the glial fibrillary acidic protein (GFAP) (rabbit polyclonal, 1:50 dilution; Bioss, Beijing, China), class III $\beta$-tubulin (TUJ-1) (rabbit polyclonal, 1:100 dilution; Abcam, Cambridge, UK), microtubuleassociated protein-2 (MAP2) (rabbit polyclonal, 1:100 dilution; Abcam, Cambridge, UK), and VIII factor (rabbit polyclonal, 1:100 dilution; Santa Cruz Biotechnology, Santa Cruz, CA, USA) at $4{ }^{\circ} \mathrm{C}$ overnight. Each 
sample was washed and then incubated with biotinylated goat anti-rabbit secondary IgG (1:200 dilution; Abcam, Cambridge, UK). These sections were processed with an avidin-biotinylated-peroxidase kit (ABC Elite kit, Burlingame, $\mathrm{CA}$ ) and mounted onto gelatin-coated glass slides. The images were observed under a 200-fold light microscope (Eclipse ET2000U, Nikon, Japan) and digitized by a slide scanner (LS-2000, Nikon Inc., Melville, NY). The immunopositive cells were counted from a sampling of 50 fields of view.

\section{TTC staining}

After the rats were sacrificed, the fresh brain sample was washed with iced PBS and placed in a brain mold for further actions. Coronal sections ( $1 \mathrm{~mm}$ in thickness) were cut throughout the cerebrum and placed in 1\% 2,3,5-Triphenyltetrazolium chloride (TTC; Sigma Chemical Co., MO, USA) for 30 mins in a $3^{\circ} \mathrm{C}$ incubator. The TTC solution was drained, and sections were immersed in $4 \%$ formalin in $0.1 \mathrm{M}$ PBS at $4^{\circ} \mathrm{C}$ and stained as previously described [14].

\section{Statistical analysis}

SPSS 24.0 software (IBM Corp., Armonk, NY, USA) was used for statistical analyses. Continuous data were presented as mean \pm standard deviation (SD), and were compared using independent sample $t$ tests. Probability $(P)$ values $\leq 0.05$ were considered significant.

\section{Results}

\section{Effects of fasudil on BMSCs in vitro}

After 24 hour treatment with fasudil, the MTT assay was performed (Fig. 1A). The OD values were 0.93 and 0.95 when BMSCs were incubated with $10 \mathrm{umol} / \mathrm{L}$, and $30 \mathrm{umol} / \mathrm{L}$ fasudil, respectively, significantly higher than the other groups studied ( $P=0.018$ and $P=0.012$, respectively). When BMSCs were incubated with $50 \mathrm{umol} / \mathrm{L}, 100 \mathrm{umol} / \mathrm{L}, 200 \mathrm{umol} / \mathrm{L}$, or $300 \mathrm{umol} / \mathrm{L}$ fasudil, the OD values were $0.70,0.55,0.44$, and 0.53 , respectively, exhibiting no difference in the $O D$ value when compared with control $(P>0.05)$. Additionally, we demonstrated that $20 \mathrm{umol} / \mathrm{L}$ fasudil could facilitate the proliferation of BMSCs and induce their differentiation into neuron-like cells. These newly differentiated cells displayed morphological trademarks such as a large and oval-shaped body with an extended projection and some short branches (Fig. 1B and C).

\section{Fluorescence observation}

The transplanted GFP-labeled BMSCs were distributed more extensively in the ischemic area (Fig. 1D) compared to the contralateral non-ischemic area (Fig. 1E). Large amounts of GFP-labeled BMSC migrated toward the lesion and survived in the neocortex, subventricular zone and corpus callosum. Moreover, most of the GFP-positive cells engrafted around the injured tissue were large and had an oval-shaped body. However, only a few cells survived in the contralateral brain. 
We collected serum samples from three rats in each group and evaluated the inflammation status by measuring the serum TNF-a levels and calculating concerning the proportion of neutrophils. The proportion of neutrophils in the fasudil+BMSC group was significantly lower than that in the BMSC alone group; the serum TNF-a level in the fasudil+BMSC group was significantly lower than that in the BMSC alone group, as well (Fig. 2, $P<0.05$ ). BMSCs increased the proportion of neutrophils 4 days after the MCAO operation; nevertheless, fasudil counteracted the increase in neutrophils. BMSCs increased the serum TNF-a levels, while fasudil exhibited the opposite effect within 5 days after the MCAO operation; ultimately, there was no significant difference in the expression of TNF-a between the fasudil+BMSC group and the control group ( $P>0.05)$. These findings indicated that fasudil could reverse the proinflammatory effects of BMSC transplantation.

\section{Immunohistochemical analysis}

The TUJ-1/MAP2/VIII factor expression in the fasudil+BMSC group was significantly higher than that in other groups $(P<0.05)$, suggesting that combined treatment may facilitate neurogenesis and angiogenesis in cerebral infarction. However, the number of GFAP-positive cells decreased dramatically in the fasudil+BMSC and BMSC alone groups $(P<0.05)($ Fig. 3$)$, indicating that the combination of BMSC and fasudil may inhibit the formation of a glial scar.

Infarct volume

Histopathological sections obtained from MCAO rats showed detectable white-patchy lesions in the areas supplied by the middle cerebral artery. TTC staining demonstrated that the lesions were distributed in the right cortex (Fig. 4B). At the multiple time points investigated, the infarct volume in the fasudil+BMSC and BMSC alone groups was significantly lower than that noted in the fasudil alone and control groups $(P<0.05)$ (Fig. 4C).

\section{Neurological function}

All rats exhibited neurological deficits one day after the MCAO operation, with mNSS scores ranging from 6 to 12 points (Fig. 4A). Three days after the MCAO operation, there was no significant difference in mNSS scores between the fasudil+BMSC group and other groups ( $P>0.05)$. After that, the mNSS scores in the fasudil+BMSC group were significantly lower than those in the other groups $(P<0.05)$. Furthermore, rats in the BMSC alone group exhibited a more remarkable improvement in neurological functions at 1 week after treatment, compared with the fasudil alone and control groups $(P<0.05)$; a difference which persisted for 3 weeks. More specifically, the functional sensorimotor recovery in the fasudil+BMSC and BMSC alone groups was more remarkable than that in the fasudil alone and control groups $(P<0.05)$. At the same time, there was no significant difference between the fasudil alone group and the control group throughout the observation period ( $P>0.05)$.

\section{Results And Discussion}


As of late, cell therapy has been proven to be an effective neurorestorative treatment for stroke, with BMSCs and human umbilical cord blood cells (HUCBCs) serving as the most commonly studied.

Evidence suggests that these stem cell-based therapies for stroke can improve neurovascular remodeling, white matter remodeling and synaptogenesis, and decrease inflammatory and immune responses [15-18]. In experimental stroke rats, BMSC treatment can decrease neuron apoptosis, improve BBB integrity, increase axonal growth and remyelination, and improve neurological functions [19,20]. Additionally, numerous studies indicate that the ROCK pathway involves cerebral ischemia, cerebral vasospasm, neural cell apoptosis, inflammation, brain microvessel function regulation, and nerve axon growth. Thus, we speculate that the combination of BMSC and fasudil may be a novel and effective regimen for treating ischemic stroke.

\section{Effects of BMSC on cerebral infarction}

Our findings demonstrated that the administration of BMSC transplantation promoted the structural and functional recovery in rats MCAO models, which is consistent with most of the previous reports. The underlying mechanisms may be multifactorial, including neuroprotective effects on focal neurons and changes in systemic homeostasis. Kuroda et al. proposed that multilineage-differentiating stress enduring (Muse) cells, isolated from the bone marrow mesenchymal cell populations, play an essential role in maintaining the living body's homeostasis may provide a plausible explanation for the neurorestorative effects of BMSCs [21]. Chau et al. found that BMSC delayed neurovascular repair and functional recovery [7]. Goldmacher et al. reported that BMSC treatment increased both neutrophil and total white blood cell counts in circulation and promoted brain astroglia and microglia activation, particularly in the infarct region [22], which is consistent with our findings. These alterations in the blood cell composition may impair the integrity of the BBB and trigger the release of cytokines and chemokines into the parenchyma.

Additionally, Chen et al. noted that BMSC treatment alone enhanced the expression of angiogenin, MMP9, and ED1 in the ischemic area [23]. These molecules are pro-inflammatory markers, which can disrupt the BBB, thereby increasing its permeability. Overall, the effects of BMSC transplantation treatment are twosided and complex; those potential benefits and, conversely, adverse effects warrant comprehensive evaluations. Xue et al. performed an extensive and systematic analysis of published controlled clinical trials, supporting that BMSC therapy is a safe and efficacious treatment for ischemia [9]. Alternatively, the development of novel strategies to attenuate those adverse effects of BMSC-induced inflammation may improve the efficacy of BMSC transplantation treatment.

\section{Effects of fasudil on ischemic stroke}

Fasudil is an isoquinoline sulfonamide derivative specifically utilized for blocking the activity of the ROCK pathway by competitively occupying the ATP binding sites [24]. Although fasudil is a selective antagonist for the Rho kinase, it can also interact with protein kinase A (PKA) and AMP-activated protein kinase (AMPK) with a low affinity, as sharing the similar structure of ATP binding sites [25]. Clinically, fasudil has been widely used to treat vasospasm and has limited use in the management of other neurological 
disorders, such as amyotrophic lateral sclerosis and spinal cord injury. Shibuya et al. performed a prospective placebo-controlled double-blind trial to evaluate the efficacy of fasudil in an acute ischemic stroke, demonstrating that fasudil administration onset significantly improved the clinical outcome [26].

Multiple mechanisms participate in the efficacy of fasudil in ischemic stroke. Firstly, Rho-kinase inhibition can increase cerebral blood flow by maintaining endothelial nitric oxide synthase [27]. Secondly, fasudil exerts neuroprotective effects by attenuating neuronal apoptosis [28]. Thirdly, fasudil may induce the differentiation of BMSCs into neuron-like cells via the Wnt/ß-catenin pathway [29]. Finally, fasudil attenuates axon growth inhibition by RhoA/ROCK in the central nervous system and thus promotes functional recovery.

Additionally, some scholars also propose that Rho/ROCK antagonists can reverse the inhibitory effects of the glial scar, thereby enhancing the neurite outgrowth [31]. Furthermore, fasudil and similar derivatives can up-regulate the production of brain-derived neurotrophic factor (BDNF) and granulocyte colony stimulating factor (GCSF), both of which are associated with neuronal differentiation and neurogenesis [32]. In the current study, we demonstrated that 20 umol/L fasudil could induce the differentiation of BMSCs into neuron-like cells portraying a large and oval-shaped morphology with a long projection and some short branches, as is consistent with the above hypotheses.

Fasudil has also been reported to protect the vascular endothelium by inhibiting neutrophil adhesion, thus exhibiting protective effects against microvascular damage, BBB disruption and inflammation [33]. In our study, fasudil treatment attenuated the accumulation of neutrophils and overexpression of TNF-a, suggesting that fasudil has anti-inflammation effects in ischemic stroke. However, we also noted that the majority of GFAP-positive cells survived, and a glial scar formed in the MCAO-sided cerebral tissues in the fasudil alone and control groups. Therefore, we speculate the neurorestorative effects of fasudil may be restricted under certain conditions; nevertheless, the definitive mechanisms require further research.

\section{Synergistic effects of BMSCs and fasudil on cerebral infarction}

The synergistic effects of BMSCs and fasudil remain unclear. Previous studies indicated that fasudil may neutralize the pro-inflammatory effects of BMSCs by down-regulating the expression of MMP9 (matrixmetalloprotease 9) [34]. In our study, BMSCs increased the proportion of neutrophils at 4 days after the MCAO operation; nevertheless, fasudil counteracted the increase of neutrophil concentration. Moreover, BMSCs increased the serum TNF-a levels, while fasudil exhibited the opposite effects within 5 days after the MCAO operation. These findings supported the above hypothesis.

Loddick et al. reported that the excessive activation of glial cells ultimately released cytokines causing damage to neurons, and formation of a glial scar inhibited the extension of the neural axon and the establishment of synaptic connections [35]. In the present study, we found that the number of GFAPpositive cells was dramatically decreased in the fasudil+BMSC and BMSC alone groups, indicating that the combination of BMSCs and fasudil can inhibit the formation of a glial scar and provide a favorable microenvironment for neurogenesis and angiogenesis. In addition, the infarct volume was noticeably 
reduced in the fasudil+BMSC group compared to the BMSC alone group, which strongly suggests that fasudil can enhance the neuroprotective effects of fasudil BMSCs in cerebral ischemia.

\section{Conclusion}

BMSCs and fasudil exert neurorestorative effects in rat models of cerebral ischemia. Fasudil neutralizes the pro-inflammatory effects of BMSCs, while BMSCs and fasudil together had synergistic effects promoting neurovascular remodeling and neurological function recovery in stroke. A combination of BMSCs and fasudil provides a promising method for the treatment of ischemic stroke.

\section{Declarations}

Ethical Approval

All procedures performed in studies involving animals were in accordance with the ethical standards of the U.K. Animals Act. This article does not contain any studies with human participants performed by any of the authors.

\section{-Consent to Participate}

Not Applicable

\section{-Consent to Publish}

All authors have the consent to publish

\section{-Authors Contributions}

All authors contributed equally

\section{-Funding}

Not applicable

\section{-Competing Interests}

There are no competition of interest

\section{-Availability of data and materials}

It is available within the manuscript

\section{References}

1. Katan, M. and A. Luft, Global Burden of Stroke. Semin Neurol, 2018. 38(2): p. 208-211.

2. Tesoro, E.P. and E. Durr, Antithrombotic Therapy for Ischemic Stroke. Neuropharmacotherapy in Critical Illness, 2018. 
3. Burman, J., et al., Autologous haematopoietic stem cell transplantation for neurological diseases. J Neurol Neurosurg Psychiatry, 2018. 89(2): p. 147-155.

4. He, $\mathrm{H}_{\text {., }}$ et al., Bone marrow mesenchymal stem cell transplantation exerts neuroprotective effects following cerebral ischemia/reperfusion injury by inhibiting autophagy via the PI3K/Akt pathway. Brain research, 2019. 1707: p. 124-132.

5. Zhang, Y., et al., Neuroprotective effects of human bone marrow mesenchymal stem cells against cerebral ischemia are mediated in part by an anti-apoptotic mechanism. Neural regeneration research, 2019. 14(4): p. 597.

6. Carpenter, A.B., et al., Understanding the Therapeutic Potential of Bone Marrow Stem Cell Therapy in Ischemic Stroke. Georgetown Medical Review, 2018. 2(1): p. 3417.

7. Chau, M.J., et al., Delayed and repeated intranasal delivery of bone marrow stromal cells increases regeneration and functional recovery after ischemic stroke in mice. BMC neuroscience, 2018. 19(1): p. 20.

8. Jiang, R.H., et al., Hypoxic conditioned medium derived from bone marrow mesenchymal stromal cells protects against ischemic stroke in rats. Journal of cellular physiology, 2019. 234(2): p. 1354-1368.

9. Xue, P., M. Wang, and G. Yan, Mesenchymal stem cell transplantation as an effective treatment strategy for ischemic stroke in Asia: a meta-analysis of controlled trials. Ther Clin Risk Manag, 2018. 14: p. 909928.

10. Sladojevic, N., B. Yu, and J.K. Liao, ROCK as a therapeutic target for ischemic stroke. Expert review of neurotherapeutics, 2017. 17(12): p. 1167-1177.

11. Kim, H.-H. and C. Ayata, Rho-Associated Kinases in Cerebrovascular Disease, in Primer on Cerebrovascular Diseases. 2017, Elsevier. p. 265-268.

12. Chiba, Y., et al., Synergistic effects of bone marrow stromal cells and a Rho kinase (ROCK) inhibitor, fasudil on axon regeneration in rat spinal cord injury. Neuropathology, 2010. 30(3): p. 241-50.

13. Mathew, B., et al., Bone-marrow mesenchymal stem-cell administration significantly improves outcome after retinal ischemia in rats. Graefe's Archive for Clinical and Experimental Ophthalmology, 2017. 255(8): p. 1581-1592.

14. Yang, M., et al., Changes in host blood factors and brain glia accompanying the functional recovery after systemic administration of bone marrow stem cells in ischemic stroke rats. Cell Transplant, 2010. 19(9): p. 1073-84.

15. Venkat, P., et al., Cell-based and pharmacological neurorestorative therapies for ischemic stroke. Neuropharmacology, 2018. 134(Pt B): p. 310-322. 
16. Garbuzova-Davis, S., et al., Intravenously Transplanted Human Bone Marrow Endothelial Progenitor Cells Engraft Within Brain Capillaries, Preserve Mitochondrial Morphology, and Display Pinocytotic Activity Toward Blood-Brain Barrier Repair in Ischemic Stroke Rats. Stem Cells, 2017. 35(5): p. 1246-1258.

17. Chen, J., et al., Neurorestorative therapy for stroke. Front Hum Neurosci, 2014. 8: p. 382.

18. Schmidt, A. and J. Minnerup, Promoting recovery from ischemic stroke. Expert Rev Neurother, 2016. 16(2): p. 173-86.

19. Li, G., et al., Bone marrow mesenchymal stem cell therapy in ischemic stroke: mechanisms of action and treatment optimization strategies. Neural Regen Res, 2016. 11(6): p. 1015-24.

20. Chen, J., P. Venkat, and M. Chopp, Bone Marrow Mesenchymal Stromal Cell Transplantation: A Neurorestorative Therapy for Stroke, in Cellular Therapy for Stroke and CNS Injuries. 2015, Springer. p. 4769.

21. Kuroda, S., et al., Muse Cell: A New Paradigm for Cell Therapy and Regenerative Homeostasis in Ischemic Stroke. Adv Exp Med Biol, 2018. 1103: p. 187-198.

22. Goldmacher, G.V., et al., Tracking transplanted bone marrow stem cells and their effects in the rat MCAO stroke model. PloS one, 2013. 8(3): p. e60049.

23. Chen, J., et al., Adverse effects of bone marrow stromal cell treatment of stroke in diabetic rats. Stroke, 2011. 42(12): p. 3551-8.

24. Sun, X.-Z., et al., Effect of Rho kinase inhibitor fasudil on the expression ET-1 and NO in rats with hypoxic pulmonary hypertension. Clinical hemorheology and microcirculation, 2019. 71(1): p. 3-8.

25. Abeysinghe, $\mathrm{H}_{\text {., }}$ et al., Modulating astrocyte transition after stroke to promote brain rescue and functional recovery: emerging targets include rho kinase. International journal of molecular sciences, 2016. 17(3): p. 288.

26. Shibuya, M., et al., Effects of fasudil in acute ischemic stroke: results of a prospective placebocontrolled double-blind trial. J Neurol Sci, 2005. 238(1-2): p. 31-9.

27. Rikitake, Y., et al., Inhibition of Rho kinase (ROCK) leads to increased cerebral blood flow and stroke protection. Stroke, 2005. 36(10): p. 2251-7.

28. Qin, T., et al., Umbelliferone reverses depression-like behavior in chronic unpredictable mild stressinduced rats by attenuating neuronal apoptosis via regulating ROCK/Akt pathway. Behavioural brain research, 2017. 317: p. 147-156.

29. Hu, Y., et al., Fasudil may induce the differentiation of bone marrow mesenchymal stem cells into neuron-like cells via the Wnt/ $\beta$-catenin pathway. Molecular medicine reports, 2019. 19(4): p. 3095-3104. 
30. He, R., et al., Effect of fasudil on cognitive function following status convulsion in rats. Molecular medicine reports, 2017. 16(1): p. 119-126.

31. Roloff, F., et al., Enhanced neurite outgrowth of human model (NT2) neurons by small-molecule inhibitors of Rho/ROCK signaling. PLoS One, 2015. 10(2): p. e0118536.

32. Li, Y.-H., et al., FSD-C10, a Fasudil derivative, promotes neuroregeneration through indirect and direct mechanisms. Scientific reports, 2017. 7: p. 41227.

33. Wang, J., et al., Fasudil inhibits neutrophil-endothelial cell interactions by regulating the expressions of GRP78 and BMPR2. Experimental cell research, 2018. 365(1): p. 97-105.

34. Knecht, T., C. Borlongan, and I. dela Peña, Combination therapy for ischemic stroke: Novel approaches to lengthen therapeutic window of tissue plasminogen activator. Brain Circulation, 2018. 4(3): p. 99.

35. Loddick, S.A., A.V. Turnbull, and N.J. Rothwell, Cerebral interleukin-6 is neuroprotective during permanent focal cerebral ischemia in the rat. J Cereb Blood Flow Metab, 1998. 18(2): p. 176-9

\section{Figures}
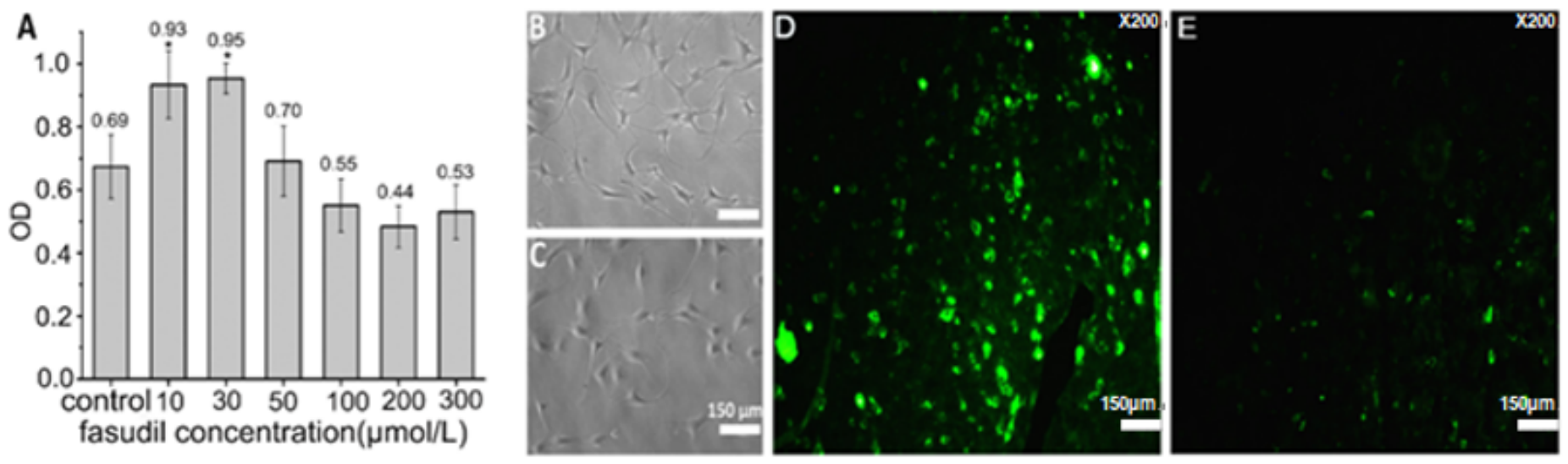

\section{Figure 1}

Effects of fasudil on BMSCs in vitro through MTT sand fluorescence observation (A) After being treated with fasudil for 24 hours, MTT assay showed $10 \mathrm{umol} / \mathrm{L}$ and $30 \mathrm{umol} / \mathrm{L}$ fasudil facilitated the significant proliferation of BMSCs $(P<0.05)$. (B\&C) Incubation with $20 \mathrm{umol} / \mathrm{L}$ fasudil induced the differentiation of BMSCs into neuron-like cells which presented a morphologically large and oval-shaped body with a long projection and some short branches. (D\&E) The transplanted GFP-labeled BMSCs distributed more densely in the ischemic area (D) when compared to the contralateral non-ischemic area (E). 
A

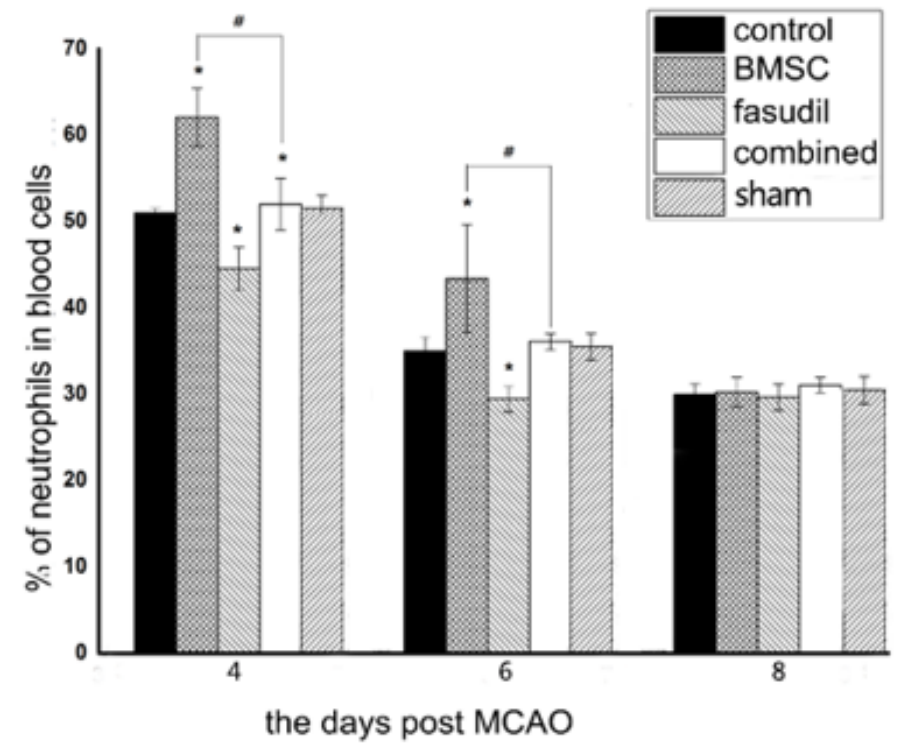

B
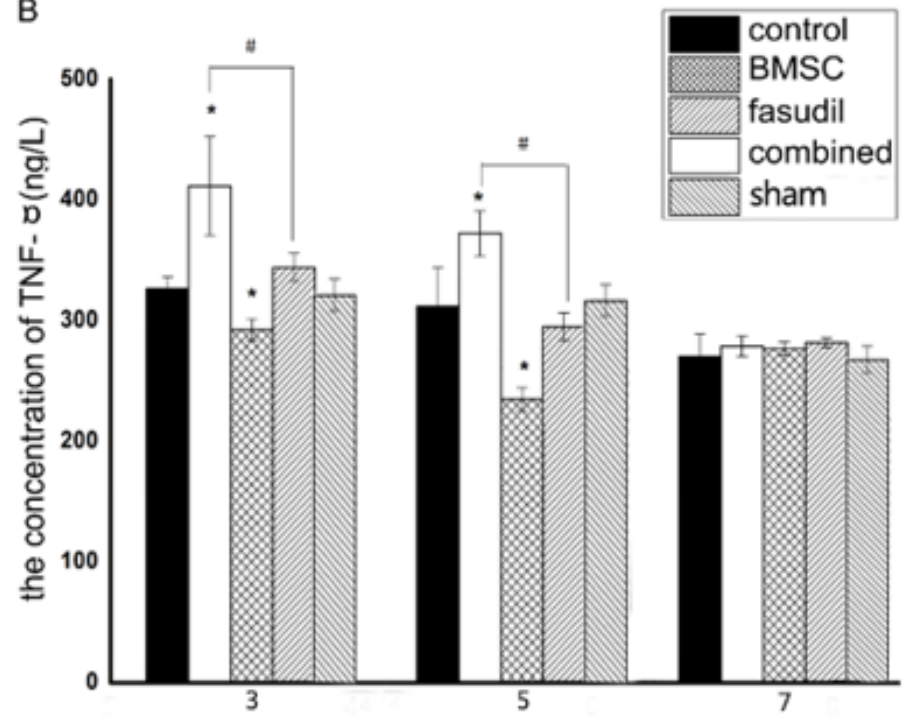

the days post treatment

Figure 2

Serum TNF-a levels and the proportion of neutrophils (A) BMSCs increased the proportion of neutrophils at 4 days after the MCAO operation; nevertheless, fasudil counteracted the increasing of neutrophils. (B) BMSCs increased the serum TNF-a levels, while fasudil exhibited opposite effects within 5 days after the MCAO operation; there was no significant difference in the expression of TNF-a between the fasudil+BMSC group and the control group $(P>0.05)$. 


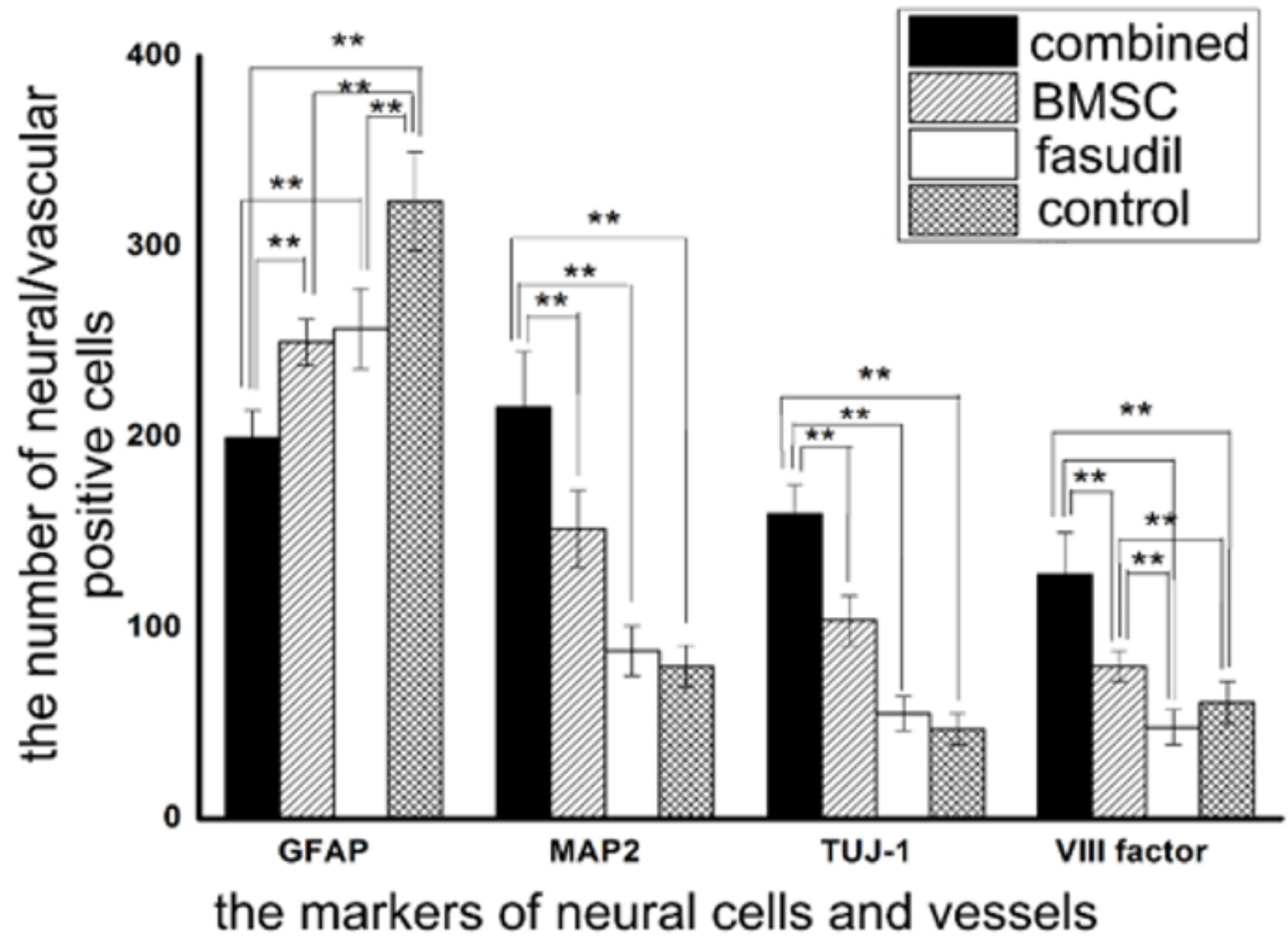

Figure 3

Statistical analysis of immunohistochemical results The expression of TUJ-1/MAP2/VIII factor in the fasudil+BMSC group was significantly higher than that in the other groups $(P<0.05)$. The number of GFAP-positive cells decreased dramatically in the fasudil+BMSC and BMSC alone groups $(P<0.05)$. 


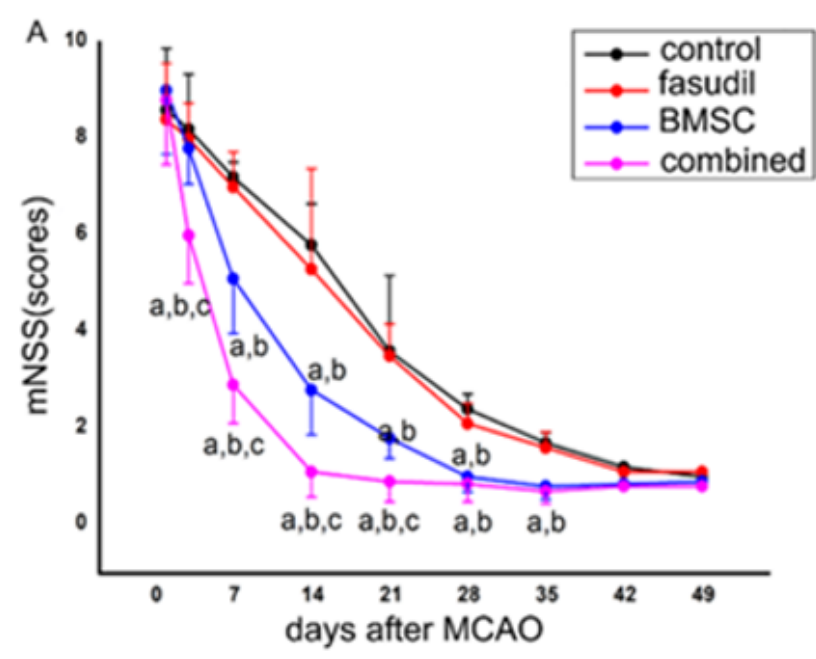

B

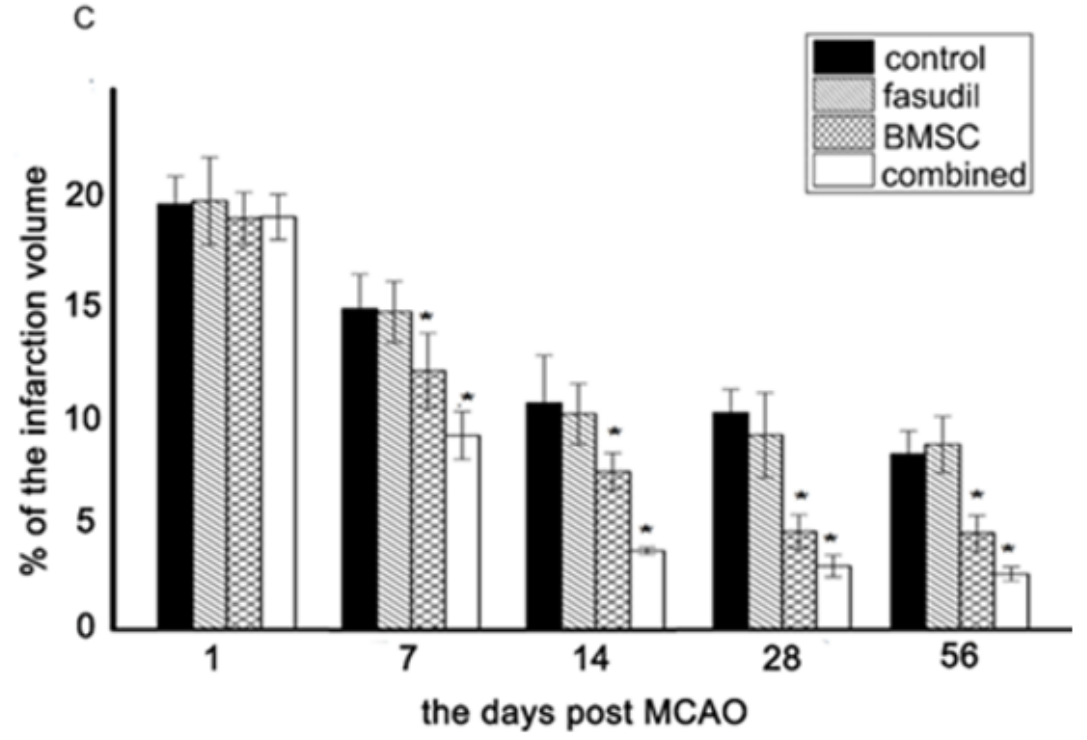

Figure 4

Neurological functional assessment and infarct volume measurement (A) Three days after the MCAO operation, the neurological function was significantly improved in the fasudil +BMSC group when compared to the other groups ( $a$, compared with the control group; $b$, compared with the fasudil alone group; c, compared with the BMSC alone group; $\mathrm{P}<0.05$ ). (B) TTC staining demonstrated that the lesions were distributed in the right cortex. (C) At the multiple time points, the infarct volume in the fasudil+BMSC and BMSC alone groups was significantly lower than that in the fasudil alone and control groups $(P<0.05)$. 